\title{
Nuevas aportaciones a la flora vascular en el País Vasco, Cantabria, Asturias y Burgos (norte de la Península Ibérica).
}

\author{
New contributions to the vascular flora of the \\ Basque Country, Cantabria, Asturias and Burgos \\ (north of the Iberian Peninsula).
}

J.A. Cadiñanos-Aguirre ${ }^{1}$, A. Llorente-Rodrigo ${ }^{2}$ y E. Fidalgo-Prieto ${ }^{2}$

$\xi^{*}$

\section{Resumen}

Se citan varios taxones interesantes de la flora vascular del Norte de la Península Ibérica, concretamente de las provincias vascas de Álava, Vizcaya y Guipúzcoa y de las comunidades de Cantabria, Asturias y Castilla-León.

Palabras clave: Spermatophyta, corología, Norte Península Ibérica, País Vasco, Cantabria, Asturias, Castilla y León.

\begin{abstract}
Several interesting taxa of vascular flora of the North of the Iberian Peninsula are recorded, specifically in the basque territories of Alava, Vizcaya and Guipuzcoa and in the communities of Cantabria, Asturias and Castile and Leon.
\end{abstract}

Key words: Spermatophyta, chorology, northern Iberian Peninsula, Basque Country, Cantabria, Asturias, Castile and Leon.

\section{Laburpena}

Iberiar penintsularen iparraldeko flora baskularraren taxon interesgarri batzuk aipatzen dira, batetik Araba, Bizkaia eta Gipuzkoa euskal herrialdeetakoak; eta, bestetik, Kantabria, Asturias eta Gaztela-Leoneko erkidegokoetakoak.

Gako hitzak: Spermatophyta, korologia, Iberiar penintsularen iparraldea, Euskadi, Kantabria, Asturias, Gaztela eta Leon.

\footnotetext{
${ }^{1}$ Departamento de Geografía, Prehistoria y Arqueología. UPV/EHU y Sociedad de Ciencias Aranzadi Correspondencia: joseantonio.cadinanos@ehu.es• joseacadi@hotmail.com

2 Sociedad Micológica y Botánica de Getxo Basozaleak Correspondencia: alfred@basozaleak.com•efidalgo@euskalnet.net. 


\section{Introducción}

El presente artículo es el resultado de reiteradas excursiones botánicas por el norte de España, de las que recogemos las citas realizadas en las siguientes provincias y/o comunidades autónomas: Álava, Asturias, Burgos, Cantabria, Guipúzcoa y Vizcaya.

Por el carácter biogeográfico del trabajo, se ha preferido en esta ocasión ordenar las plantas por criterios corológicos. De esta manera se han agrupado los taxones en los siguientes grupos corológicos:

- Elemento eurosiberiano que comprende al eurosiberiano en sentido estricto, así como al atlántico, al boreo-alpino y al pirenaico-cantábrico, incluyendo los endemismos con distribución principal en esta zona.

- Elemento mediterráneo que se refiere al mediterráneo en sentido amplio así como al mediterráneo montano y al mediterráneo-atlántico; también a los endemismos ibéricos y a algunos arqueófitos circunmediterráneos.

- Otros elementos corológicos u otros orígenes: agrupa el elemento circumboreal, plurirregional y a las plantas introducidas, naturalizadas, etc.

\section{Material y métodos}

Habitualmente conservamos los pliegos de las plantas en los herbarios particulares de los autores (J.A. Cadiñanos: Arangu-Angi-; E. Fidalgo: EFP; Alfred Llorente: ALF), salvo de aquellas plantas de determinación segura y/o cuya recolección, a nuestro juicio, podría suponer un riesgo para la pervivencia de la nueva población encontrada. En tal caso, hemos sustituido el material de herbario por fotografías tomadas en el propio lugar del hallazgo y/o en estudio (de manera, que donde no hacemos referencia a herbario, siempre hay una fotografía como soporte gráfico). En todos los casos, se anotan con suficiente precisión las coordenadas UTM tomadas con GPS y algunas notas ecológicas y geográficas.

Para la nomenclatura nos hemos basado principalmente en Flora Iberica (Castroviejo et al., 1980-2014) tanto de los volúmenes publicados, como de los borradores disponibles en línea. Para la corología, además de consultar también dicha flora, hemos tomado como fuente principal Aizpuru et al. (1999) dado que el marco de estudio de nuestro trabajo está centrado en el norte peninsular; también hemos consultado otras fuentes (Villar, Sesé \& Ferrández, 1997 y 2001), en especial cuando dicho taxón no aparece en la obra de Aizpuru et al. Dentro de cada apartado, las plantas aparecen ordenadas alfabéticamente. Es un hecho reseñable también que recientemente, gracias a su autor, Juan Antonio Durán, y a su editor, José Luis Benito, por fin contamos con el catálogo de la flora vascular de Cantabria (Durán, 2014), que tan necesario era y que se ha convertido inmediatamente en una inmejorable herramienta para comprobar si nuestras citas de esta comunidad tienen algún valor corológico. 


\section{Resultados y discusión}

\section{ELEMENTO EUROSIBERIANO}

(comprende los elementos eurosiberiano, orófito europeo, atlántico y boreo-alpino y los endemismos cantábricos y/o pirenaicos e ibéricos atlánticos o subatlánticos).

\section{Carex pulicaris $L$.}

SS, Oiartzun, subida a Aiako Harriak, 30TWN9497, 52 m, cuneta con humedad permanente sobre suelo ácido, 23.05.2013, A. Llorente. Herbario personal ALF no 2013-05-23 (2).

Con esta nueva localización complementamos las aportadas para Guipúzcoa por Aseginolaza et al. (1985: 1093) en Deba, Orio, Aia, Sierra de Aralar y Sierra de Alzania; por Willkomm (1893: 29-30) y Bubani (1901: 215) en Hernani; y por Allorge \& Allorge (1941: 236) y Catalán \& Aizpuru (1985: 83) en Jaizkibel.

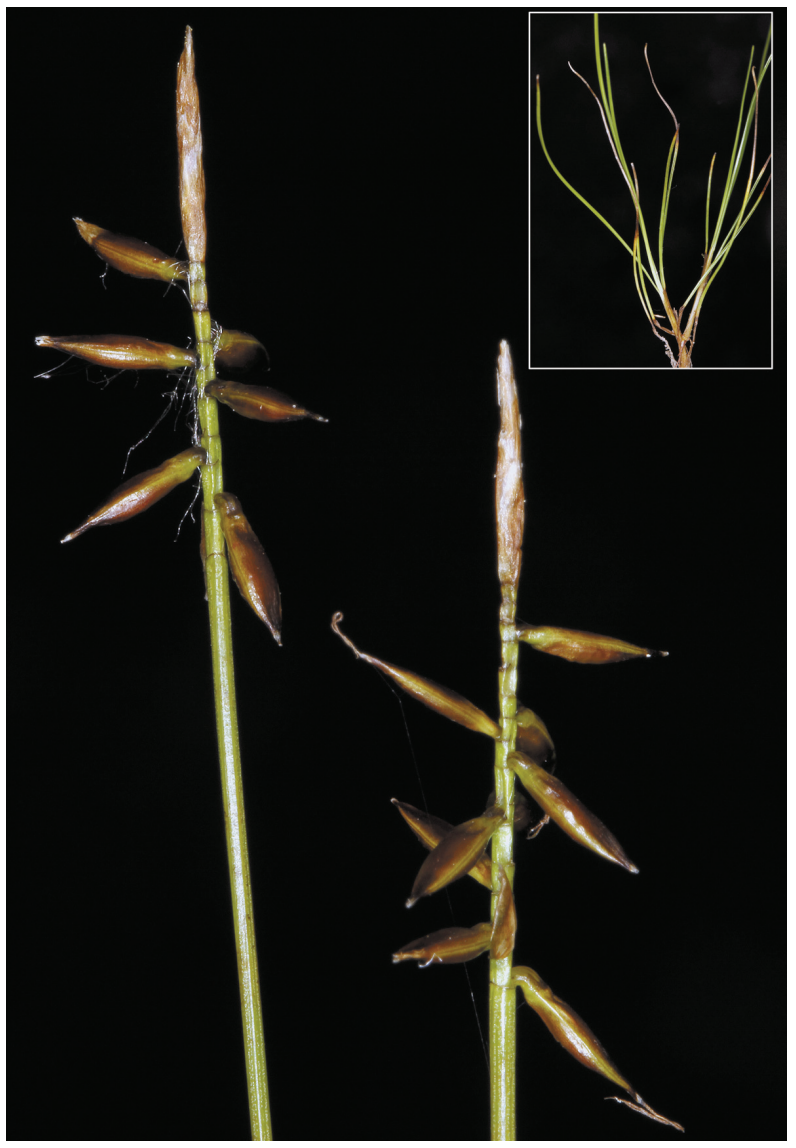

Fig. 1.- Carex pulicaris, Aiako Harriak (Oiartzun) 


\section{Colchicum gr. autumnale L.}

Bi, Carranza, Aldeacueva, 30TVN6882, 511 m, cunetas herbosas de camino forestal, 23.09.1995, A. Llorente. Herbario personal ALF no 1995-09-23 (1).

Bi, Arcentales, San Miguel de Linares, 30TVN8188, 345 m, 20.09.1997, A. Llorente. Herbario personal ALF no 1997-09-20 (1).

Bi, Carranza, La Escrita, 30TVN7488, 435 m, borde de camino, 14.09.2012, A. Llorente. Herbario personal ALF no 2012-09-14 (6).

Aportamos estas citas para la provincia de Vizcaya, que se suman a las de Aseginolaza et al. (1985: 904) en Traslaviña, Onaindia (1986: 199) en Upo (Gorbea), Pérez de Ana (2004: 71) en La Calera del Prado y Patino et al. (2010: 96) en Sopelana y Concha, todas como C. autumnale. Pero, dada la alta complejidad que muestra este género, nosotros hemos preferido citarlas como grupo autumnale siguiendo las cautelas expuestas por Rico (2013), aunque, según los estudios moleculares, lo más probable es que nuestras citas vizcaínas se correspondan realmente con Colchicum lusitanicum Brot.

\section{Convallaria majalis L.}

S, Castro Urdiales, Talledo, 30TVN8695, 320 m, alfombra briofítica sobre argilitas en bosque de frondosas, 25.08.2013 y 10-V-2014, J.A. Cadiñanos.

Se trata de una exigua población de esta lilácea, que suele mostrar querencias más montanas, (en Durán, 2014: 308, su altitud mínima para Cantabria es de 1000 m) de ahí su interés. Población a preservar de la que estamos haciendo seguimiento.

Crepis paludosa (L.) Moench.

Bu, Espinosa de los Monteros, Puerto Estacas de Trueba, 30TVN4274, 1145 m, pastos acidófilos húmedos a orillas de arroyos y encharcamientos, 31.07.2012, J.A. Cadiñanos, E. Fidalgo y A. Llorente. Herbario personal ALF no 2012-07-31 (10).

Alejandre et al. (2006: 192) advierten de la escasez de esta compuesta en la provincia y que tiene un alto requerimiento de humedad edáfica. Anotamos, pues, una localidad más para el extremo septentrional de Burgos, próxima a las encontradas por Herrera (1995: 81) y Loidi et al., (2014: 63).

Doronicum carpetanum Boiss. y Reuter ex. Willk. subsp. pubescens (C. Pérez Morales, A. Penas, F. Llamas y C. Acedo) Aizpuru

S, Castro Urdiales, Callejamala, 30TVN8392, 510 m, cabecera de regato en umbría, 17-V2009, J.A. Cadiñanos y A. Llorente. Herbario personal ALF no 2009-05-17 (12).

S, Castro Urdiales, Talledo, 30TVN8695, 300-320 m, megaforbios en cabeceras de arroyos y en canteras abandonadas, 23.04.2014, J.A. Cadiñanos. Herbario personal Arangu-Angi14042301. 
S, Castro Urdiales, Baltezana, 30TVN8697, 88 m, aliseda con megaforbios, 20.05.2014, J.A. Cadiñanos.

Seguimos encontrando en Castro Urdiales nuevas poblaciones, más o menos nutridas, de esta espectacular y llamativa compuesta, que ya citábamos anteriormente del alto de las Muñecas (Cadiñanos, Llorente \& Fidalgo, 2005: 44). El ambiente es también similar al reseñado: comunidades de megaforbios en cabeceras de arroyos, canteras o minas a cielo abierto abandonadas... siempre en umbrías. La población de Baltezana estaba constituida, que sepamos, por un solo ejemplar del que, por supuesto, no se recogió muestra.

\section{Epipactis helleborine (L.) Crantz subsp. helleborine}

Bi, Sopelana, Zalduondo, 30TWP00, 50 m, orla de encinar cantábrico, 19-V-2006, E. Fidalgo y A. Llorente, Herbario personal ALF no 2006-05-19 (2).

Bi, Muskiz, San Julián, 30TVN9097, 50 m, seto de encinas sobre margas, 22.06.2013, J.A. Cadiñanos. Herbario personal Arangu-Angi-13062201.

Siempre resulta interesante encontrar nuevas poblaciones de taxones de orquídeas que no son precisamente muy comunes en la Cornisa Cantábrica, en especial por la cada vez menor presencia de hábitats adecuados. En este caso se trata de poco más que un ribazo de encinas en el margen de las mieses de la solana del Pico Ramos, apenas un ínfimo resto de los encinares que debieron cubrir la zona; pese a ello sirve de refugio a algunas plantas raras y/o de sesgo mediterráneo. Recientemente dicha mies, que constituía un paisaje bastante singular y valioso en Vizcaya y además había llegado relativamente intacta hasta nuestros días (eso sí, como prados), ha resultado muy alterada por nuevas construcciones totalmente disonantes.

\section{Epipactis phyllanthes G.E. Smith}

Bi, Muskiz, Borja, 30TVN9094, 108 m, cuneta umbrosa y húmeda de antiguo ferrocarril, 5.07.2013, M.A. Bernaola y E. Fidalgo. Herbario personal n¹30701.

Hace unos años (Fidalgo, Llorente \& Cadiñanos, 2005: 157-158) citamos E. phyllanthes de las dunas de Górliz siguiendo la nomenclatura de Flora Iberica (Crespo, 2005) en lo que era la segunda población peninsular conocida de la especie. En el caso que nos ocupa, hemos encontrado tres ejemplares de pequeño tamaño, con la misma morfología que presentaban las plantas de nuestra cita anterior.

\section{Erucastrum nasturtiifolium subsp. sudrei Vivant}

Bi, Sopuerta, Pico Cinto, 30TVN8887, 495 m, umbría herbosa sobre margocalizas, 5.07.2014, J.A. Cadiñanos y A.Llorente. Herbario personal Arangu-Angi-14070501.

Encontramos una pequeña población de esta crucífera en unos espolones margocalizos situados en la ladera norte de esta modesta alineación montañosa. En este ambiente umbroso se refugian también otras plantas no muy comunes en el conjunto de la comunidad. 


\section{Galium verum L. subsp. verum}

Bi, Galdames, Campa de la Loca-Gardújulo, 30TVN8887, 470 m, pastizal ralo sobre margocalizas, 30.06.2012, J.A. Cadiñanos y E. Fidalgo. Herbario personal Arangu-Angi-12063001.

No resulta una planta muy abundante en los montes y valles atlánticos y, en Vizcaya, sólo existe una cita anterior de Górliz (Aseginolaza et al., 1985: 571) más cercana al mar que ésta, de ahí también su interés.

\section{Geranium sanguineum $L$.}

S, Castro Urdiales, monte San Pelayo, 30TVP8102, 145 m, herbazal en lapiaz, 9.05.2009, J.A. Cadiñanos.

S, Castro Urdiales, Ontón, 30TVN8599, 46 m, herbazal entre rocas calizas, 20.05.2014, J.A. Cadiñanos. Herbario personal Arangu-Angi-14052003.

Nos parecen interesantes estas citas de un geranio no demasiado común o al menos no demasiado citado. La población del karst de monte Pelayo es relativamente abundante y de aparición regular, en el sentido de que, en años posteriores, la hemos visto con asiduidad.

\section{Gymnadenia conopsea (L.) R. Br.}

Bi, Sopuerta, Luchaco, 30TVN8786, 380 m, talud herboso sobre margas, 30.06.2012, J.A. Cadiñanos y E. Fidalgo. Herbario personal Arangu-Angi-12063005.

Otra orquídea de exigua aparición en Vizcaya y cuyas citas anteriores son, por lo general, de zonas situadas más al interior o a mayor altitud, como las de Aseginolaza et al. (1985: 1100) de El Mazo (Carranza), Orduña, Aldamin-Gorbea, Txarlazo-Sierra Salvada y Urkiola.

\section{Hypericum undulatum Schousb. ex Willd.}

Bi, Erandio, Goierri, 30TWN0396, 40 m, cuneta de carretera secundaria en ambiente húmedo de robledal acidófilo, 23.07.2006, A. Llorente. Herbario personal ALF n 2006-0723 (5).

S, Castro Urdiales, Fuentelateja, 30TVP7903, 380 m, manantial y ladera rezumante, 30.07.2012, J.A. Cadiñanos. Herbario personal Arangu-Angi-12073001.

Hipérico ligado a sitios húmedos que no aparece muy citado en Vizcaya, aunque es casi seguro que resulta más abundante pero localizado. Así, aunque en Flora Ibérica (Castroviejo et al., 1993: 234) no la anotan para la provincia de Vizcaya, sí existían citas anteriores en Bakio (Dupont, 1964), Somorrostro (Aseginolaza et al., 1985: 446) y en el embalse de Lertutxe, Leioa (Biurrun \& López, 1991: 71). 2

Lathyrus niger (L.) Bernh.

Bu, Valle de Mena, Concejero, 30TVN7172, 440 m, quejigal-robledal, 14.07.2012, J.A. Cadiñanos y E. Fidalgo. Herbario personal Arangu-Angi-12071401. 


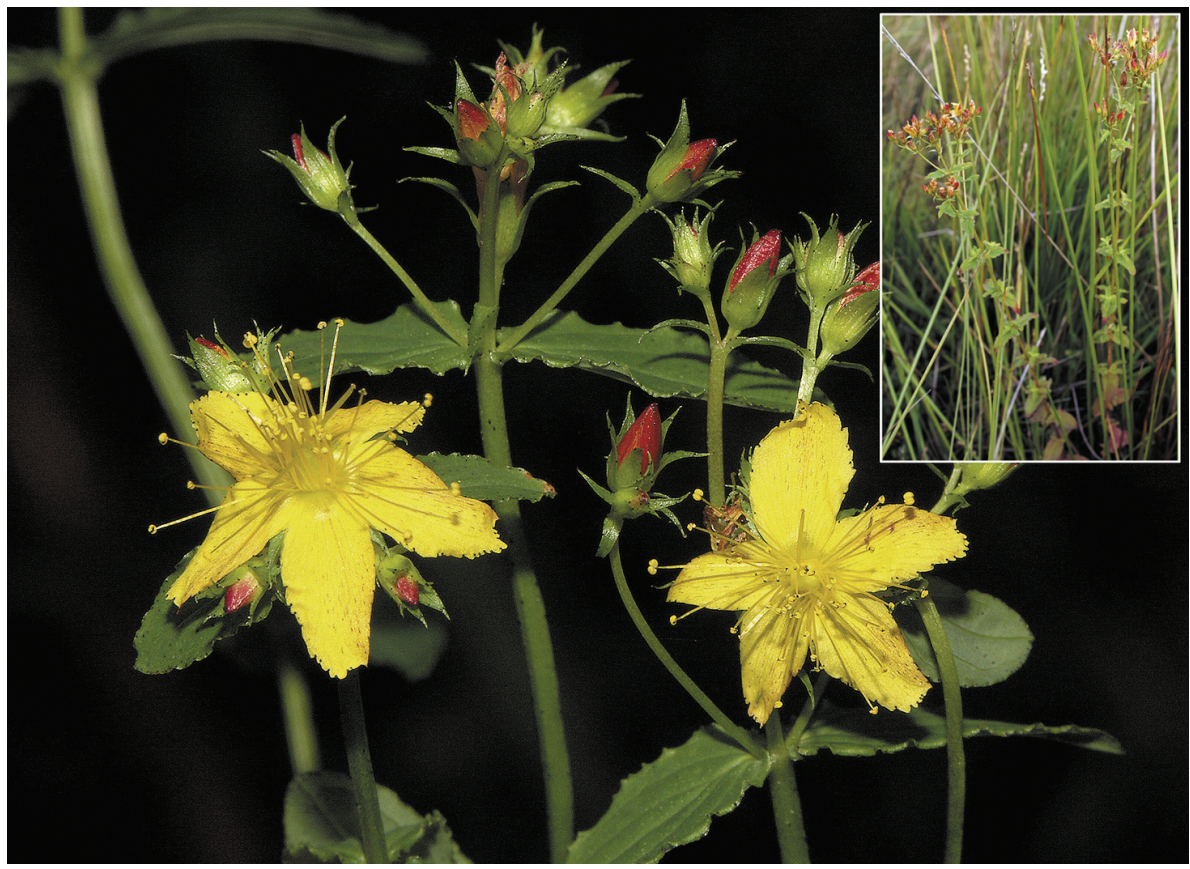

Fig. 2.- Hypericum undulatum, Goierri (Erandio) y, la foto pequeña, de Fuentelateja (Castro Urdiales)

Fig. 2.- Hypericum undulatum, Goierri (Erandio) and, small figure Fuentelateja (Castro Urdiales)

A pesar de su corología eurosiberiana apenas existen citas al norte de la divisoria de aguas mediterráneo-cantábrica, si exceptuamos la de Herrera (1995: 117). En Burgos, no nos consta ninguna anterior, ya que Alejandre et al. (2006: 406) sólo la han encontrado en la zona media y sur de la provincia.

\section{Lathyrus sylvestris $L$.}

Bi, Galdames, El Escobal, 30TVN9089, 153 m, cunetas y setos, 25.07.2013, J.A. Cadiñanos. Herbario personal Arangu-Angi-13072501.

S, Castro Urdiales, Cerdigo, 30TVP7805, 50 m, cuneta húmeda, 17.07.2013, J.A. Cadiñanos. Herbario personal Arangu-Angi-13071701.

No hay apenas citas anteriores de la zona baja comprendida entre Cantabria y Vizcaya para esta planta que cada vez encontramos con más frecuencia (Llorente, Cadiñanos y Fidalgo, 2009a: 56; Durán, 2014: 158; Aseginolaza et al., 1985: 340; Anthos, 2014).

\section{Lilium pyrenaicum Gouan}

Bi, Lemoiz, Arminza, 30TWP0809, 10-30 m, herbazal junto a carretera y playa de cantos, 21.05.2008, J.A. Cadiñanos y K. Ugartetxe. 


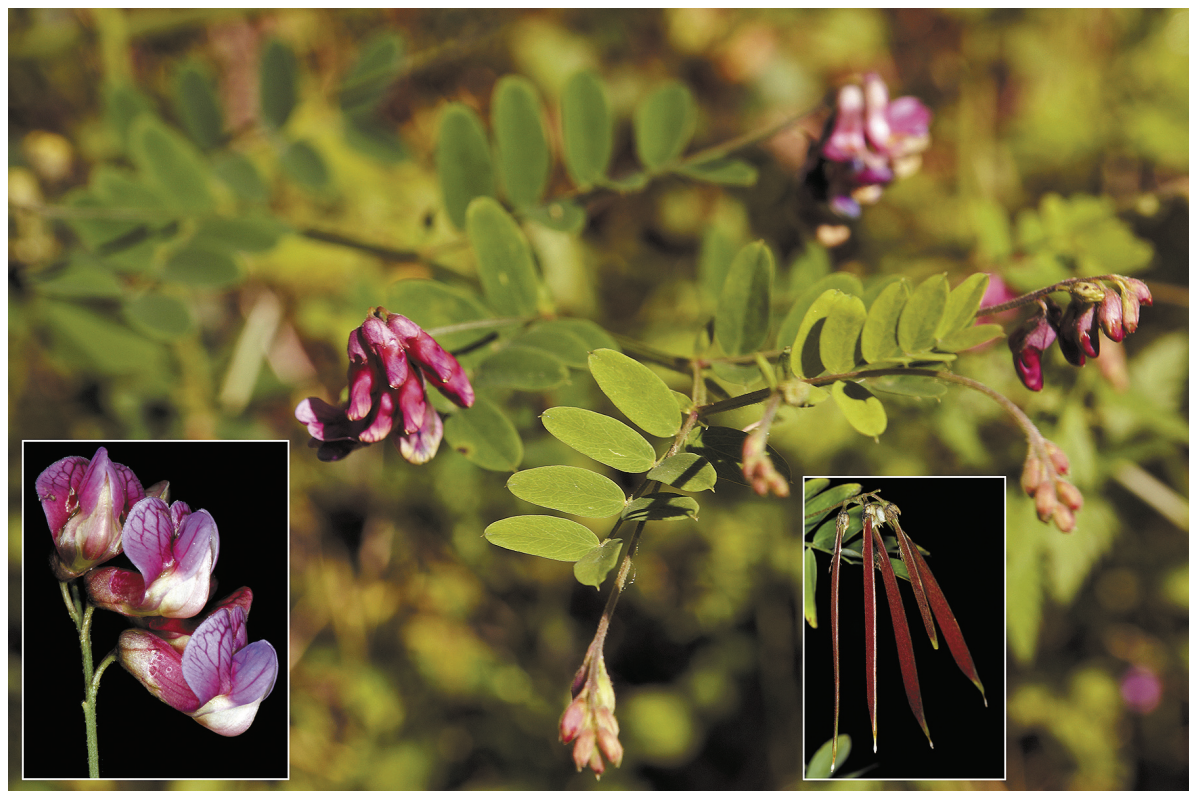

Fig. 3.- Lathyrus niger, Concejero (Valle de Mena)

S, Castro Urdiales, monte San Pelayo, 30TVP8102, 150 m, herbazal en lapiaz, 24.05.2014, J.A. Cadiñanos y A. Llorente.

S, Castro Urdiales, ensenada de Urdiales, 30TVP8104, 7 m, lapiaz herboso a borde del mar, 8.06.2014, J.A. Cadiñanos y C. Melgosa.

Esta especie habita desde el nivel del mar hasta cotas alpinas, tanto en substratos calizos como silíceos, pero siempre en zonas con humedad edáfica. Juzgamos interesantes estas nuevas citas. No se recogieron muestras por lo escaso de las poblaciones y por su inconfundible aspecto, aunque sí se hicieron fotografías georreferenciadas.

\section{Onobrychis viciifolia Scop.}

SS, Irún, polígono industrial de Araso, 30TWN9497, 52 m, orilla de la carretera en la misma rotonda de acceso al polígono Araso y también en herbazales sobre terrenos removidos en la carretera de acceso principal, 17.05.2013 y 3.06.2013, A. Llorente. Herbario personal ALF no 2013-05-17 (1).

Aunque en Castroviejo et al. (2000: 966) no se menciona para la provincia de Guipúzcoa, Aseginolaza et al. (1985: 383) anotaban en cambio su presencia en Lasarte, considerándola rara para la vertiente cantábrica. Aizpuru et al., (2005: 77) la citan de Bergara y Aretxabaleta. Aportamos, por tanto, una nueva cita provincial, cuyo origen puede deberse al uso forrajero de esta planta, así como en las hidrosiembras y restauraciones vegetales en los taludes de las carreteras. 
Campos \& Herrera (2009: 111) la consideran como una especie alóctona naturalizada no invasora. Aunque no especifican las localidades, los puntos marcados en el mapa parecen referirse a los lugares indicados anteriormente.

\section{Scilla lilio-hyacinthus L.}

Bi, Sollube, Meñaka, Arroyo Sangrazilo, 30TWP1803, 344 m, orilla del arroyo, 11.03.2009, A. Llorente. Herbario personal ALF no 2009-3-11 (3).

S, Castro Urdiales, Baltezana, 30TVN8697, 88 m, aliseda con megaforbios, 20.05.2014, J.A. Cadiñanos.

Estas poblaciones destacan, una vez más, por la escasa altitud a la que se ubican, en especial la de Baltezana-Castro Urdiales que seguramente es de las más bajas de toda la Cornisa Cantábrica. Por lo reducido de esta población no se recogió muestra ya que además se trata de una especie que, en estos ambientes y en esta zona, no admite confusión.

\section{Senecio nemorensis $L$.}

Bi, Berango, Larrañazubi, 30TWP0299, 19 m, población numerosa con alta humedad edáfica en un robledal, 19.08.2013, M.A. Bernaola y E. Fidalgo. Herbario personal no EFP140803. O, Ribadesella, Sardalla, 30TUP3112, 74 m, manantial en la linde con un bosque mixto de Quercus robur, Castanea sativa, etc., 15.08.2013, E. Fidalgo y L. Makazaga. Herbario personal no EFP140801.

O, Llanes, Mere, 30TUP4401, 284 m, manantial junto al río Bedón, en cuarcitas, 16.08.2013, E. Fidalgo y L. Makazaga. Herbario personal no EFP140802.

O, Santiago, 30TTP5700, 100 m, cuneta de carretera en suelo encharcado en la proximidad del río Trubia, 7-VIII-2014, I. Borlán, A. Llorente y S. Llorente. Herbario personal ALF no $2014-$ 08-07 (2).

Citado por Aseginolaza et al. (1985: 795) en Urkiola y Otxandiano, nuestra cita de Vizcaya es la más cercana al litoral en esta comunidad junto con la de Bermeo de Aparicio et al. (1994: 97). Respecto a Asturias hay escasas citas, dos en el interior occidental (Rodríguez Guitán, 2010) y una en la zona central costera (Aedo, 2003), todas ellas alejadas de las que aportamos ahora.

\section{Succisa pratensis Moench}

Bi, Galdames, El Escobal, 30TVN9088, 150 m, cabecera sombría y húmeda de un prado, 21.10.2011, J.A. Cadiñanos y M. Gómez. Herbario personal Arangu-Angi-11102101.

Bi, Arcentales, Laiseca, 30TVN8287, 210 m, prado húmedo, 04.01.2014, J.A. Cadiñanos. Herbario personal Arangu-Angi-14010401.

S, Liendo, El Cerrillo, 30TVP6803, 105 m, prado juncal, 14.11.2013, J.A. Cadiñanos. Herbario personal Arangu-Angi-13111401. 
En la parte central de Las Encartaciones de Vizcaya y en la Cantabria oriental seguramente es una planta más común en los prados húmedos de lo que reflejan las escasas citas incluidas hasta la fecha en Anthos (2014).

\section{Stachys alpina L.}

Bi, Gatika, 30TWP10, 80 m, campas sobre suelos pedregosos calizos, 1.05.2002, A. Llorente. Herbario personal ALF no 2002-05-01 (11).

Bi, Zalla, Las Fuentes, 30TVN8986, 380 m, robledal en suelo pedregoso-margoso, 19.04.2006, J.A. Cadiñanos.

Bi, Mungia, 30TWP1400, 45 m, terreno removido y nitrificado, 9.05.2009, A. Llorente. Herbario personal ALF no 2009-05-09 (19).

Bi, Urduliz, La Campa, 30TWP0202, 50 m, claro en encinar sobre flysh margoso, Población muy copiosa, 25.02.2012, J.A. Cadiñanos y E. Fidalgo.

Bi, Sopuerta, Pico Cinto, 30TVN8887, 502 m, claro herboso en margocalizas, 30.06.2012, J.A. Cadiñanos y E. Fidalgo. Herbario personal Arangu-Angi-12063006.

S, Castro Urdiales, depósito de aguas, 30TVP8102, 201 m, gleras de karst, 10.04.2010, J.A. Cadiñanos y A. Llorente. Herbario personal ALF no 2010-04-10 (10).

S, Castro Urdiales, Ontón, 30TVP8600, 30 m, ruderal, parte superior herboso del acantilado, 24.05.2014, J.A. Cadiñanos y A. Llorente. Herbario personal Arangu-Angi-14052401.

No nos parece rara esta planta en el litoral comprendido entre Cantabria y Vizcaya, aunque algunas fuentes opinen lo contrario y la tengan como escasa para la zona (Aseginolaza et al., 1985: 622; Durán, 2014: 147; Anthos, 2014). Más bien habría que calificarla como localizada porque requiere de lugares frescos sobre suelos no muy ácidos o neutrobásicos, circunstancias de concurrencia limitada en este contexto.

\section{Trifolium strictum $\mathrm{L}$.}

Vi, Izkiz, Quintana, 30TWN4324, 765 m, pastos arenosos que se encharcan temporalmente, 13.07.2013, A. Llorente. Herbario personal ALF no 2013-07-13 (11).

Flora Iberica (Castroviejo et al., 2000: 701) no menciona su presencia para Álava, como tampoco lo hace Aseginolaza et al. (1985: 399). En Anthos (2014) sólo existen citas para Treviño, en la provincia de Burgos. Las únicas citas para Álava son del Puerto de Vitoria y de Bajauri (Treviño) y fueron aportadas por Alejandre et al. (1987: 126). La nuestra se convierte así en la tercera localidad para esta provincia.

Tripleurospermum inodorum (L.) Sch. Bip.

(M. maritima L. subsp. inodora (C. Koch) Soó)

Bi, Górliz, Andramari, 30TWP0506, 35 m, alcorque junto al polígono industrial, 9.09.2010, E. Fidalgo. 


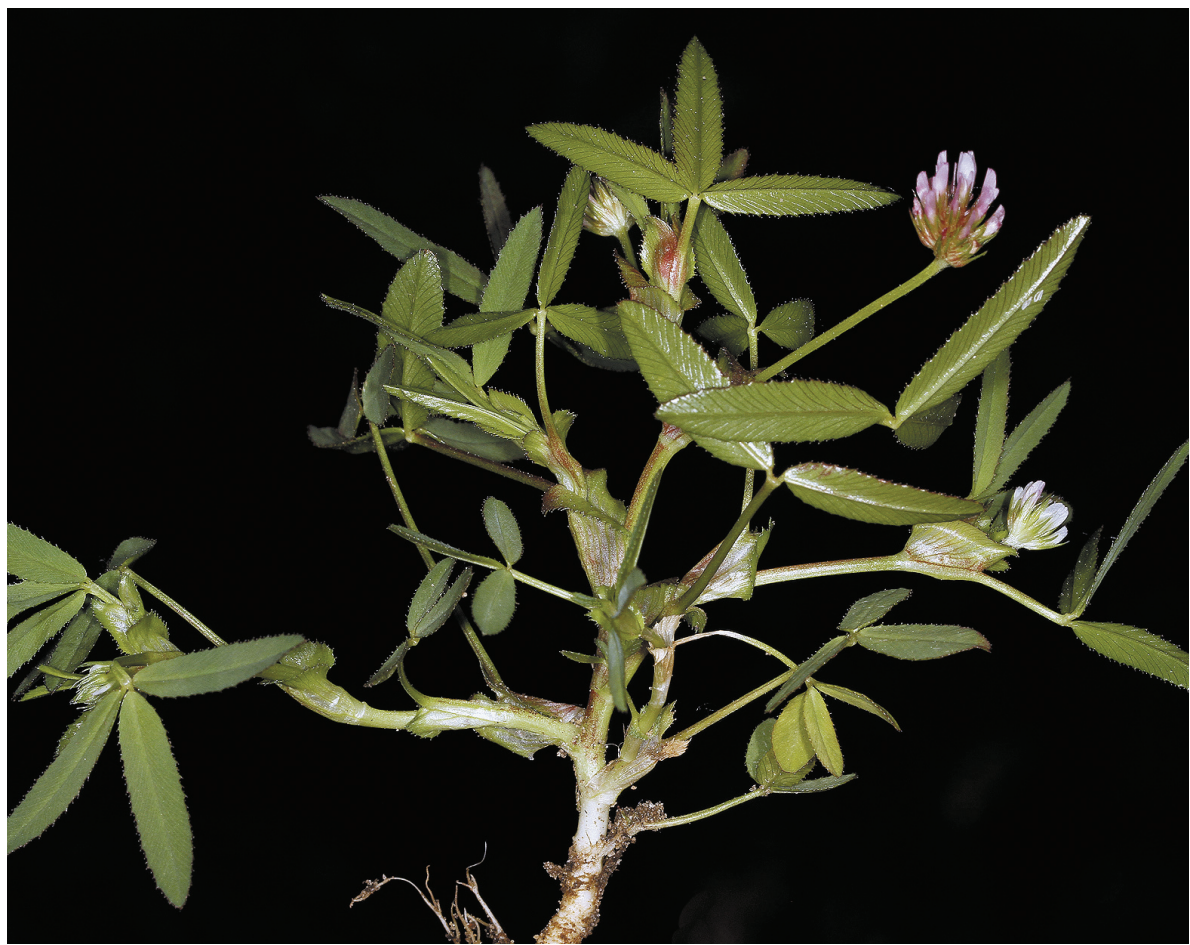

Fig. 4.- Trifolium strictum, Quintana (Izkiz)

Bi, Getxo, Ibarberango, 30TVP9901, 8 m, rotonda bajo la autopista, 7.07.2013, E. Fidalgo. Herbario personal ALF n²014-06-28 (14).

Rara compuesta en nuestro territorio que Aseginolaza et al. (1985: 779) dieron a conocer sólo para Álava (Lasarte y Lapuebla de Labarca). Posteriormente, Aizpuru et al. (1989-90: 125 y 1998: 17) la citaron, como Matricaria perforata Merat, en Legorreta y Donostia, en Guipúzcoa, donde ya era conocida de Beasain (Braun-Blanquet, 1967). Ahora aportamos como novedad provincial dos localidades para Vizcaya.

\section{Verbascum lychnitis L.}

Bu, Espinosa de Los Monteros, Valnera-Bernacho, 30TVN4777, 1080 m, ruderal en borde de pista, 5.08.2013, J.A. Cadiñanos. Herbario personal Arangu-Angi-13080501.

Resulta extraño que no aparezca más citada o sólo de zonas más meridionales en Alejandre et al. (2006: 615), pues comprobamos que es planta abundante en la zona mencionada. Precisamente, en fecha un poco anterior a la nuestra (7.08.2013), Antonio González Gómez la cita también de Espinosa de Los Monteros (Herbario Digital Xabier de Arizaga), aunque a menor altitud (728 m) y más cerca del núcleo urbano. 


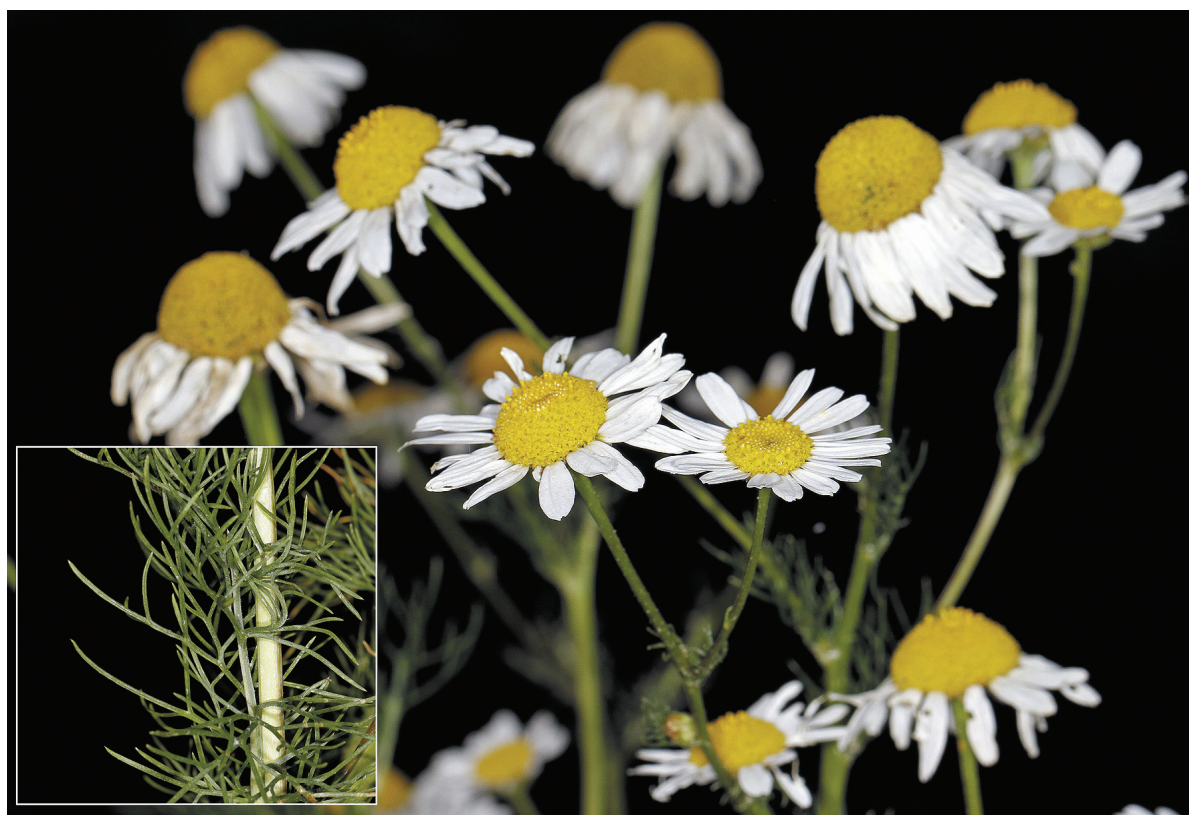

Fig. 5.- Tripleurospermum inodorum, Ibarberango (Getxo)

\section{Viola cornuta L.}

Bu, Espinosa de los Monteros, La Sía, 30TVN5377, 1145 m, cabecera de arroyo, 15.06.2013, J.A. Cadiñanos y E. Fidalgo. Herbario personal Arangu-Angi-13061503.

Primera cita de esta violeta para Burgos ya que no aparece citada ni en la primera flora provincial de Alejandre et al., 2006, ni en la actualización posterior (Alejandre et al., 2014); además resulta interesante porque completa un significativo vacío en su distribución cantábrica conocida hasta la fecha, que discurría desde las poblaciones picoeuropeanas al oeste (las más cercanas, en Piedras Luengas) hasta las prepirenaicas de Aralar por el este.

\section{Xiphion latifolium Miller}

(= Iris latifolia (Miller) Voss)

S, Castro Urdiales, monte San Pelayo, 30TVP8102, 150 m, herbazal en lapiaz, 26.05.2013 y 06.08.2013, Carlos Melgosa. Herbario personal Arangu-Angi-13080601.

Una nueva e interesante localidad de este lirio que, junto a las ya conocidas de Las Encartaciones de Vizcaya: Musques, Ciérvana, Galdames y Barakaldo (Aseginolaza et al., 1985: 936; Pérez de Ana, 2004: 72; Patino et al., 2010: 99), se ubica a medio camino entre las más abundantes poblaciones de Pirineos y de la Cordillera Cantábrica. 


\section{ELEMENTO MEDITERRÁNEO}

(incluye los elementos mediterráneo, mediterráneo-atlántico y orófito mediterráneo y los endemismos ibérico-mediterráneos).

\section{Aegilops ventricosa Tausch.}

Bu, Santo Domingo de Silos, 30TVM6446, 1004 m, borde de camino en herbazal húmedos sobre suelo removido, 13.06.2012, A. Llorente. Herbario personal ALF no 2012-06-13 (31).

Bu, Cebrecos, 30TVM5146, 1001 m, pastos, 4.06.2014, A. Llorente. Herbario personal ALF no 2014-06-04 (32).

A las escasas citas de esta gramínea aportadas por Alejandre et al. (2006: 712) para la mitad meridional de la provincia burgalesa, sumamos las nuestras, que suponen un cierto enlace geográfico entre las de Burgos capital y la Sierra de Neila.

\section{Arisarum simorrhinum Durieu}

S, Castro Urdiales, Ontón, 30TVN8599, 51 m, entre pastos y rocas calizas, 20.05.2014, J.A. Cadiñanos.

Pequeña población cobijada en una hondonada entre calizas y margas de flysh; es posible que haya algunas más en similares condiciones, incluso a mayor altitud. Se añade a otras poblaciones ya referidas, como Arisarum vulgare subsp. transiens, de Castro Urdiales (Aedo et al., 2001: 41), una de ellas, en el puente de Ontón sobre el río Sabiote, seguramente muy cercana a la nuestra, pero que no parece ser la misma, ya que está a mayor altitud. No se recogió muestra, dada su excepcionalidad en el ámbito cantábrico y su fácil identificación.

Bombycilaena erecta (L.) Smolj.

Bi, Sopuerta, Pico Cinto, 30TVN8887, 473 m, descarnaduras, repisas y escarpes erosionados en margocalizas, 30.06.2012, J.A. Cadiñanos y E. Fidalgo. Herbario personal Arangu-Angi12063001.

En los montes costeros vizcaínos hay citas anteriores en Punta Lucero y Monte Serantes (Onaindia, 1986:194) y de otros intermedios como Monte Alén y Arraiz de Patino (2011: 95), a los que se suma esta población de una planta que no deja de ser escasa y valiosa en este ámbito.

\section{Brachypodium distachyon (L.) P. Beauv.}

Bi, Sopuerta, Pico Cinto, 30TVN8887, 470 m, descarnaduras y escarpes erosionados en margocalizas, 30.06.2012, J.A. Cadiñanos y E. Fidalgo. Herbario personal Arangu-Angi-12063002. Gramínea de distribución mediterránea y de pastos efímeros que apenas alcanza los valles o montes atlánticos de Vizcaya, salvo en Monte Serantes o Algorta según Aseginolaza et al. (1985: 996). 


\section{Bupleurum baldense Turra}

Bi, Sopuerta, Pico Cinto, 30TVN8887, 470 m, descarnaduras y escarpes erosionados sobre margocalizas, 30.06.2012, J.A. Cadiñanos y E. Fidalgo. Herbario personal Arangu-Angi12063003.

S, Castro Urdiales, alto de San Andrés, 30TVP8104, 35 m, pastos efímeros sobre margas, 7.06.2005, J.A. Cadiñanos, E. Fidalgo y A. Llorente.

Umbelífera terófita de pequeño tamaño (por lo que suele pasar desapercibida) de distribución mediterránea occidental, rara en la Cornisa Cantábrica, pese a lo cual hay unas cuantas citas previas de Vizcaya a cargo de Aseginolaza et al. (1985: 504) de Monte Lucero, Monte Serantes, Bilbao, Górliz, Orduña y Mendiola; así como de Cantabria por Durán (2014: 244) que recoge las localidades citadas hasta la fecha, siendo la más próxima la de Monte Candina, Liendo (Herrera, 1995: 65).

Carlina corymbosa L. subsp. major (Lange) J. López y Devesa

Bi, Gorliz, Fanos, 30TWP0508, 195 m, prado en acantilado, 6.09.2013, E. Fidalgo. Herbario personal no 120801

López \& Devesa (2010: 191-192) presentan esta nueva combinación y la citan en Vizcaya, pero sin concretar el lugar.

\section{Chaenorhinum serpyllifolium (Lange) Lange subsp. serpyllifolium}

VI, Valdegovia, Tuesta, 30TVN 9839, 551 m, pastos secos y arenosos en borde de camino, 25.08.2012, I. Borlán, A. Llorente y S. Llorente. Herbario personal ALF no 2012-08-25 (7).

Aseginolaza et al. (1985: 661) la citan para Álava en Fontecha, Comunión, Monte Cabrera, Salinillas de Buradón y Elciego. Aportamos nuestra cita del valle de Valdegovia, dando continuidad geográfica en su distribución hacia Burgos: Valle de Tobalina y Miranda de Ebro (Alejandre et al., 2006: 595).

Dactylis glomerata subsp. hispanica (Roth) Nyman

Bi, Sopuerta, Pico Cinto, 30TVN8887, 470 m, descarnaduras y escarpes erosionados sobre margocalizas, 30.06.2012, J.A. Cadiñanos y E. Fidalgo. Herbario personal Arangu-Angi12063004.

Añadimos una nueva cita a las anteriores de Aseginolaza et al. (1985: 978) y de Loidi et al. (1997) para este taxón mediterráneo, no muy común en Vizcaya.

\section{Linum trigynum $\mathrm{L}$.}

Bi, Galdakao, Erletxe, 30TWN1687, 100 m, prado ajardinado, 2.07.2013, E. Fidalgo. Herbario personal ALF no 2013-06-26 (1). 


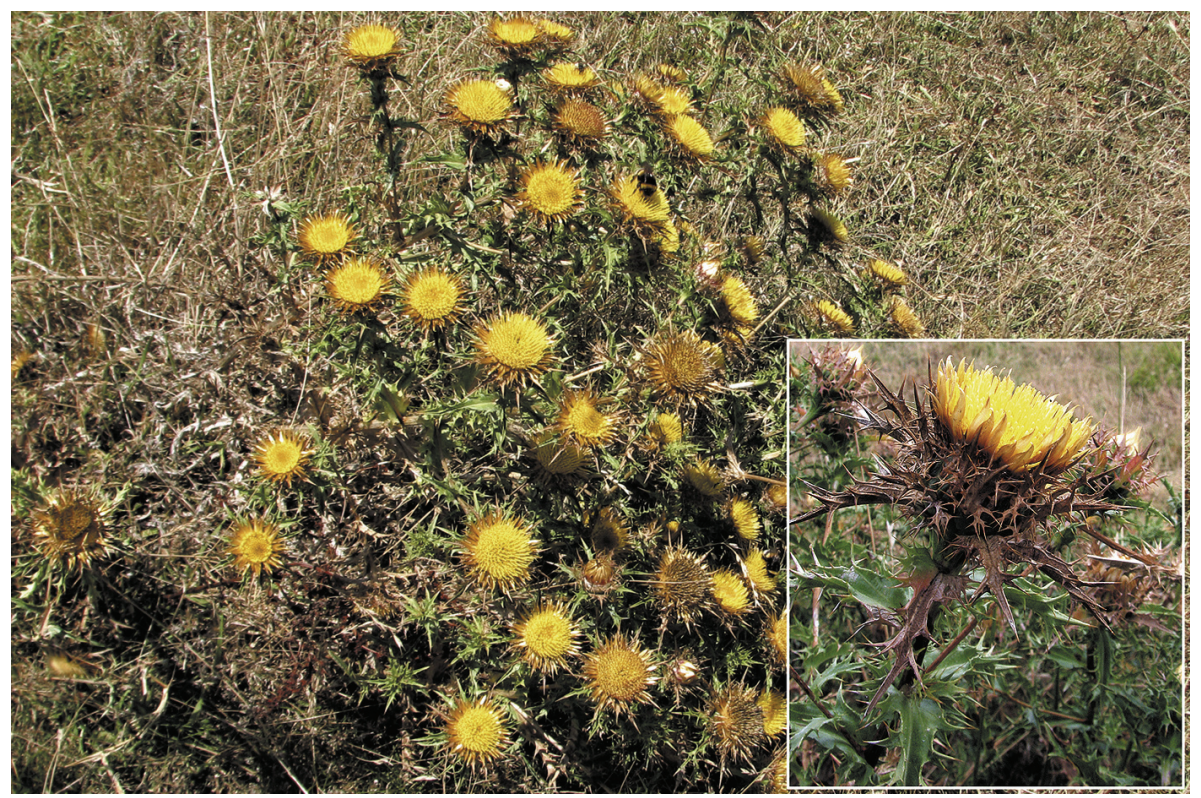

Fig. 6.- Carlina corymbosa subsp. major, Fanos (Gorliz)

Bi, Gorliz, Urezarantza, 30TWP0507, 100 m, prado sobre margas, 19.07.2013, J.A. Cadiñanos y E. Fidalgo. Herbario personal no 130701.

Estas citas darían continuidad espacial a las reseñadas por Aseginolaza et al. (1985: 398) de Monte Lucero y de la playa de Laga.

\section{Lotus angustissimus L.}

Vi, Izkiz, Quintana, 30TWN4324, 765 m, pastos-juncales arenosos que se encharcan temporalmente, 13.07.2013, A. Llorente. Herbario personal ALF no 2013-07-13 (9).

La ausencia de citas alavesas en Aseginolaza et al. (1985: 374) se subsanó con las aportadas por Urrutia \& Zorrakin (1986: 213), en Larrintxo (Korres), Bajauri y Obekuri, y por Campos et al. (2002: 128) en Bideko (Amurrio). Nos parece conveniente añadir nuestra cita para un mejor conocimiento de su distribución en esta provincia.

\section{Melilotus segetalis (Brot.) Ser.}

Bi, Sopelana, Bareño, 30TWP0103, 54 m, terreno removido pendiente de edificar, 15.05.2013, E. Fidalgo. Herbario personal ALF no 2013-05-18 (2).

Citada por Aseginolaza et al. (1985: 352) en Unbe (Vizcaya). Aizpuru et al. (1999: 317) la describen como muy rara y de terrenos removidos, como en nuestro caso. Es planta alóctona (Herrera \& Campos, 2010: 44). 


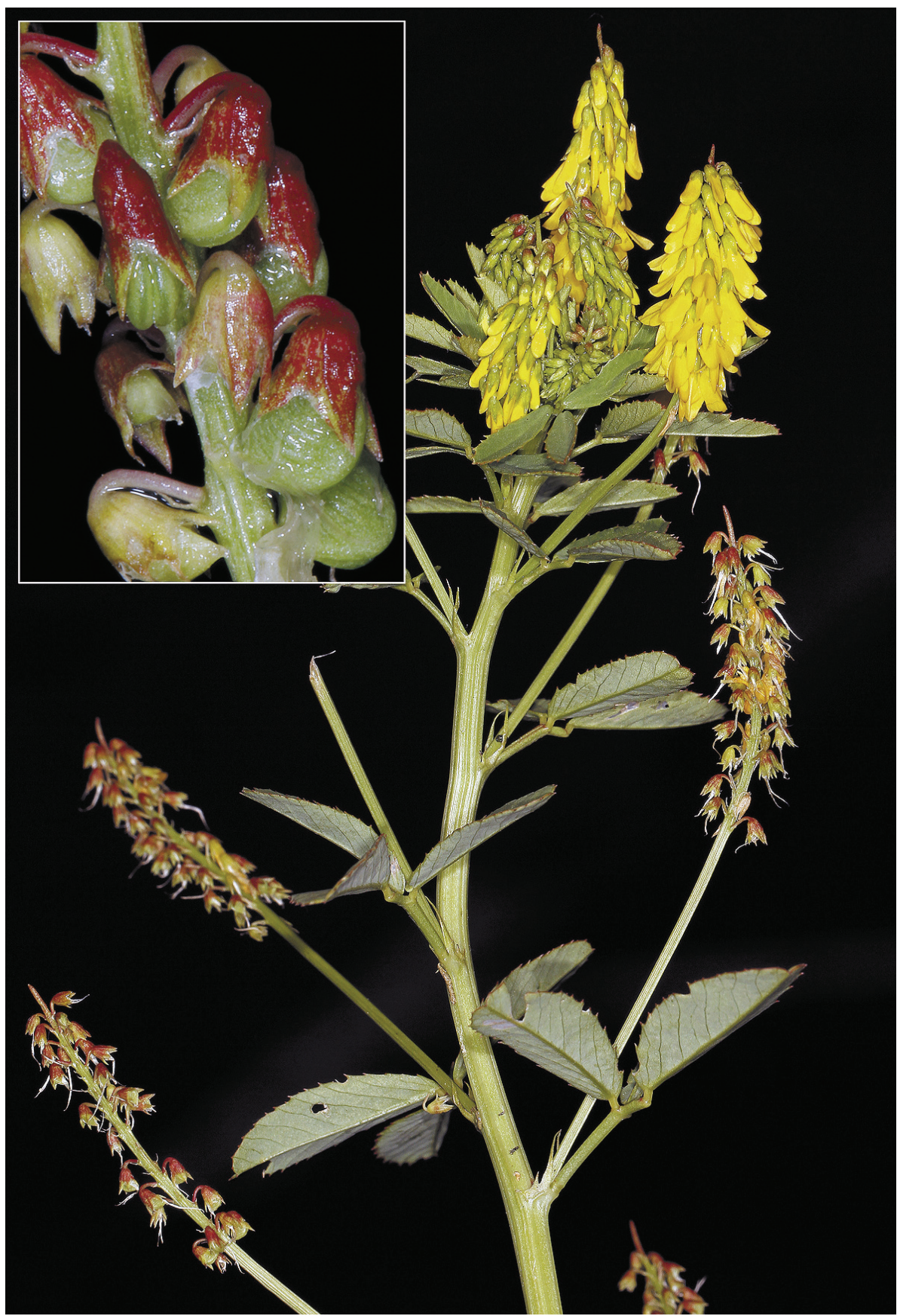

Fig. 7.- Melilotus segetalis, Bareño (Sopelana) 
Romulea clusiana (Lange) Nyman

S, Santoña, playa de Berria, 30TVP6112, 4 m, dunas terciarias, 30.03.2013, J.A. Cadiñanos y C. Melgosa. Herbario personal Arangu-Angi-13033001.

Recientemente citada también de las dunas de Trengandin (Noja), Cantabria (Carlón et al., 2013: 115) ésta sería la segunda para la región. Al parecer se está expandiendo de oeste a este por los sistemas dunares cantábricos, ya que existen también referencias recientes de Asturias. Como afirman los autores antes citados, este endemismo de los arenales de las costas atlánticas ibéricas y marroquíes -que al parecer solo se insinúa levemente en la costa mediterránea malagueña y en contadas localidades de la submeseta sur-supone novedad cántabra casi inmediatamente después de haberlo sido para Asturias -cf. Porto Torres (2013: 4)-, y en conjunto ve desplazarse 300 km hacia el este un área en el noroeste peninsular cuyo extremo, como quedo dicho, bien creímos estar señalando con mucha aproximación al hacer la cita de la Marina lucense que figura en Aedo et al. (2001: 93).

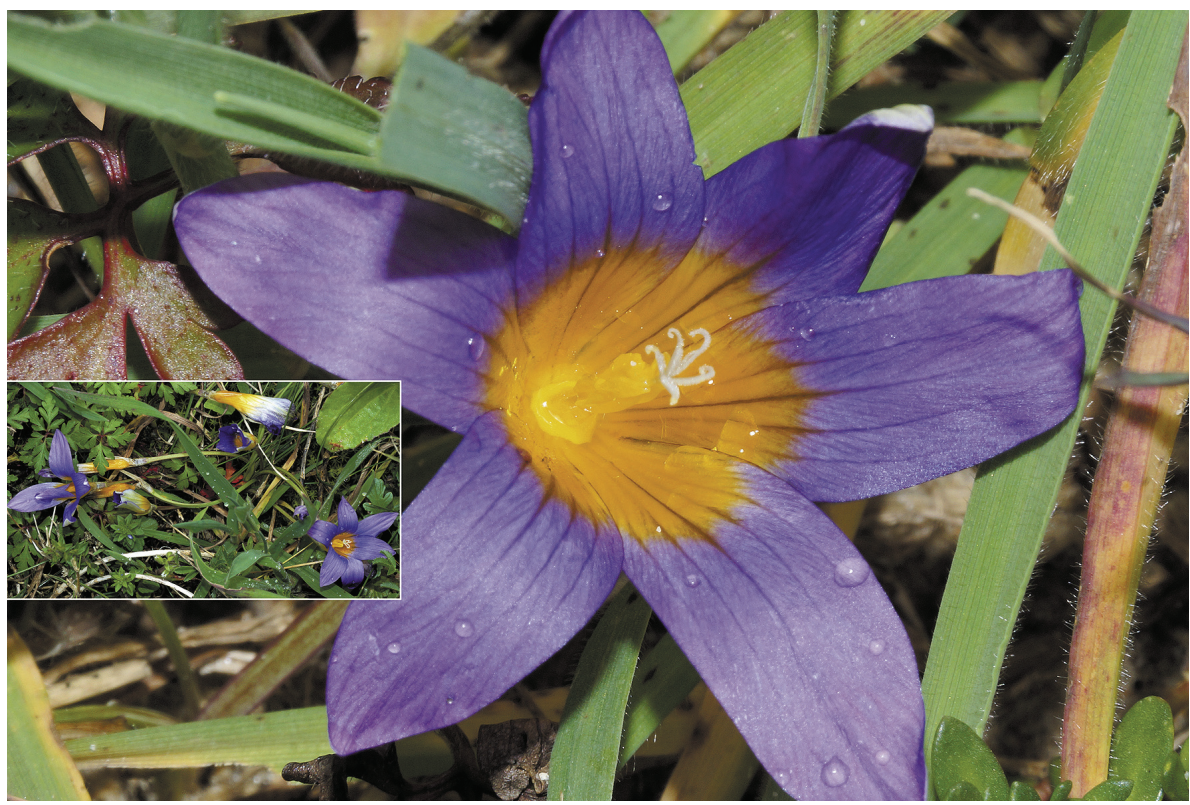

Fig. 8.- Romulea clusiana, Playa de Berria (Santoña)

\section{Ruta chalepensis L.}

Bi, Sopuerta, Alcedo, 30TVN8689, 169 m, prado en calizas, 16.03.2013, J.A. Cadiñanos y A. Llorente.

Citada ya para esta provincia en Bilbao por Willkomm (1880: 516), en Lekeitio por Guinea (1949: 199) y en el monte Serantes por Onaindia (1986: 222). 
Esta planta termófila se enrarece hasta desaparecer tan pronto nos alejamos de la línea costera, por ello encontramos relevante esta cita del interior de Vizcaya, en Sopuerta, no lejos de unos caseríos, por lo que quizás esté naturalizada, como así la consideran Campos \& Herrera (2009: 118).

\section{Senecio lividus $L$.}

Bu, Espinosa de los Monteros, Puerto Estacas de Trueba, 30TVN4274, 1149 m, suelos ácidos someros en ambiente de brezales y turberas, 31.07.2012, J.A. Cadiñanos, E. Fidalgo y A. Llorente. Herbario personal ALF no 2012-07-31 (14).

Alejandre et al. (2006: 240) sólo aportan media docena de localidades disyuntas en la provincia de Burgos para esta pequeña compuesta, lo que justifica nuestra aportación.

\section{Scabiosa atropurpurea $\mathrm{L}$.}

S, Castro Urdiales, Allendelagua, 30TVP8004, 40 m, cuneta entre dos viales, 23.08.2013, J.A. Cadiñanos. Herbario personal Arangu-Angi-13072301.

Faltan citas previas del oriente cántabro de una planta que cada vez resulta más común en viales, eriales, etc., de la zona litoral entre Cantabria (comunidad de donde Durán 2014: 119- sólo la recoge de la franja costera occidental) y Vizcaya (Aseginolaza et al., 1985: 733).

\section{OTROS ELEMENTOS COROLÓGICOS}

\section{(subcosmopolita, plurirregional, circumboreal e introducidas)}

\section{Gymnocarpium dryopteris (L.) Newman}

S, Soba, sierra de Hornijo-Hoyo Salzoso, 30TVN5488, 910 m, umbría en roquedo calizo, 15.06.2013, J.A. Cadiñanos y E. Fidalgo. Herbario personal Arangu-Angi-13061501.

Helecho de preferencias montanas ya citado de las cercanas montañas pasiegas (Durán, 2014: 29) y, a 1200 m, en el portillo de La Sía (Herrera, 1995: 106), pero no nos constan citas anteriores de la sierra del Hornijo.

\section{Linum usitatissimum $\mathrm{L}$.}

S, Castro Urdiales, Ontón, 30TVP8600, 40 m, ruderal, 3.06.2013, J.A. Cadiñanos. Herbario personal Arangu-Angi-13060301.

Se trata de una pequeña población probablemente adventicia al estar situada al borde de una pista cerca de la autovía A8. Durán (2014: 170) recoge dos citas anteriores de Cantabria. 


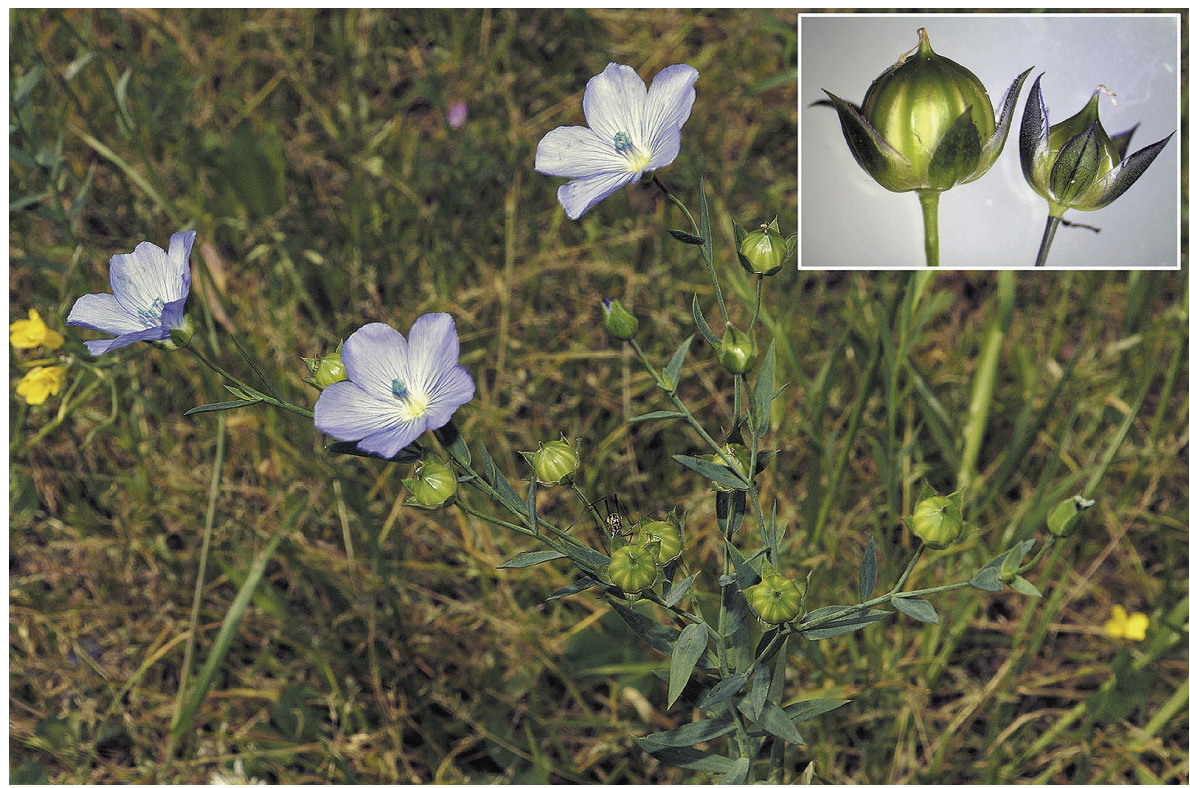

Fig. 9.- Linum usitatissimum, Ontón (Castro Urdiales). En la foto pequeña, a la derecha, cápsula de L. bienne.

Fig. 9.- Linum usitatissimum, Ontón (Castro Urdiales). In the small figure, right, capsule L. bienne.

Lotus hispidus Desf. ex DC.

Bi, Sopelana, 30TWP0203, 70 m, talud junto a un camino, 13.06.2008, E. Fidalgo. Herbario personal no 080601.

Bi, Berriatua, 30TWN4497, 90 m, 20.06.2011, talud junto a la carretera del acantilado, E. Fidalgo.

Bi, Urdaibai, Murueta, 30TWP2501, 2 m, herbazal en borde de marisma, 25.05.2012, A. Llorente. Herbario personal ALF no 2012-05-25 (3).

Para Vizcaya, Aseginolaza et al. (1985: 373) aportaron las localidades de Monte Ogoño (Elantxobe), Gernika y Balmaseda como Lotus subbiflorus Lag. subsp. subbiflorus, y Guinea (1953: 355) la de Urberuaga de Ubilla, denominándola Lotus hirsutus Desf. Se amplía así las nuestras el área de distribución para esta provincia.

\section{Monotropa hypopitys L.}

Bi, Carranza, La Calera del Prado, 30TVN6580, 560 m, sobre la hojarasca de hayedo, 22.06.2002, A. Llorente. Herbario personal ALF no 2002-06-22 (1).

Bi, Laukiniz, Talleri, 30TWP0600, 55 m, sotobosque de Quercus robur, 29.06.2002, A. Llorente. 
Bu, Merindad de Cuesta Urria, Mijangos, 30TVN6542, 556 m, sotobosque arenoso de pinar marítimo (Pinus pinaster), 20.06.2010, A. Llorente. Herbario personal ALF no 2010-06-20 (14).

En Vizcaya, Aseginolaza et al. (1985: 531) sólo aportaron dos citas en Pando (Carranza) y Orduña. De Orduña también la ha mencionado Pérez de Ana (2008: 93) y (2010: 63). Patino \&Valencia (1989: 81) la refieren de Itxina (Gorbea). Respecto a Burgos, nuestra localización complementa a las aportadas por Alejandre et al. (2006: 456) para la mitad septentrional provincial. Bajo nuestro criterio, la importancia de estas nuevas ubicaciones reside, no sólo en procurar un mejor conocimiento de su distribución para ambas provincias, sino también en el hecho de haberlas encontrado en ambientes menos típicos para esta especie micoheterótrofa (pinar arenoso y robledal), que sobre la hojarasca de hayedo donde es más habitual o, al menos, donde la hemos visto con más frecuencia.

\section{Orobanche amethystea Thuill.}

Bi, Getxo, La Galea, 30TVP9701, 70 m, con Eryngium campestre en landa costera, 16.06.2012, E. Fidalgo. Herbario personal EFP no120601.

Bi, Abanto, La Balastera, 30TVN9295, 125 m, lateral de la antigua vía del tren a Galdames, acompañando a Hypochoeris radicata, 03.07.2013, E. Fidalgo. Herbario personal n०130801. La cita más cercana es la de GBC (2014) para Castro Urdiales y de Onaindia (1986: 216) en Monte Serantes. Anthos (2014) recoge una cita de Laínz (1960) para Renteria en Guipúzcoa.

\section{Reseda lutea L. subsp. lutea}

S, Castro Urdiales, Ontón, 30TVP8600, 30 m, ruderal, parte superior herboso del acantilado, 20.05.2014, J.A. Cadiñanos. Herbario personal Arangu-Angi-14052001.

Pequeña población refugiada en la cabecera, un tanto ruderalizada, de un acantilado en antigua zona minera. Al parecer esta planta es rara en la Cornisa Cantábrica y en la Cantabria más oriental en particular, de donde Durán (2014: 202) no la menciona, de ahí su interés.

\section{Silene latifolia Poiret}

Ss, Oiartzun, Aiako Harriak, 30TWN9595, 314 m, herbazal ruderalizado en cuneta, 29.04.2013, A. Llorente. Herbario personal ALF no 2013-04-29 (4).

Este hallazgo corrobora su presencia en el extremo oriental guipuzcoano, complementando las aportadas por Catalán \& Aizpuru (1985: 32) en Hondarribia y Pasai Donibane, citadas como Silene alba, y por Biurrun et al., (2011: 72) en Zaldibia. No conocemos más localidades provinciales.

\section{Sisyrinchium rosulatum E.P. Bicknell}

Bi, Muskiz, monte Mello, 30TVN8994, 95 m, cunetas herbosas de camino forestal, 23.05.2009, J.A. Cadiñanos, R. Fernández, E. Fidalgo y C. Monedero. Herbario personal ALF no 2013-07-12 (1). 
Se trata de una especie originaria del SE de Norteamérica que, en España, Castroviejo et al. (2014: 452-454), sólo anotan su presencia en las provincias de Huelva y Pontevedra. Por tanto, nuestra cita supone novedad para Vizcaya.

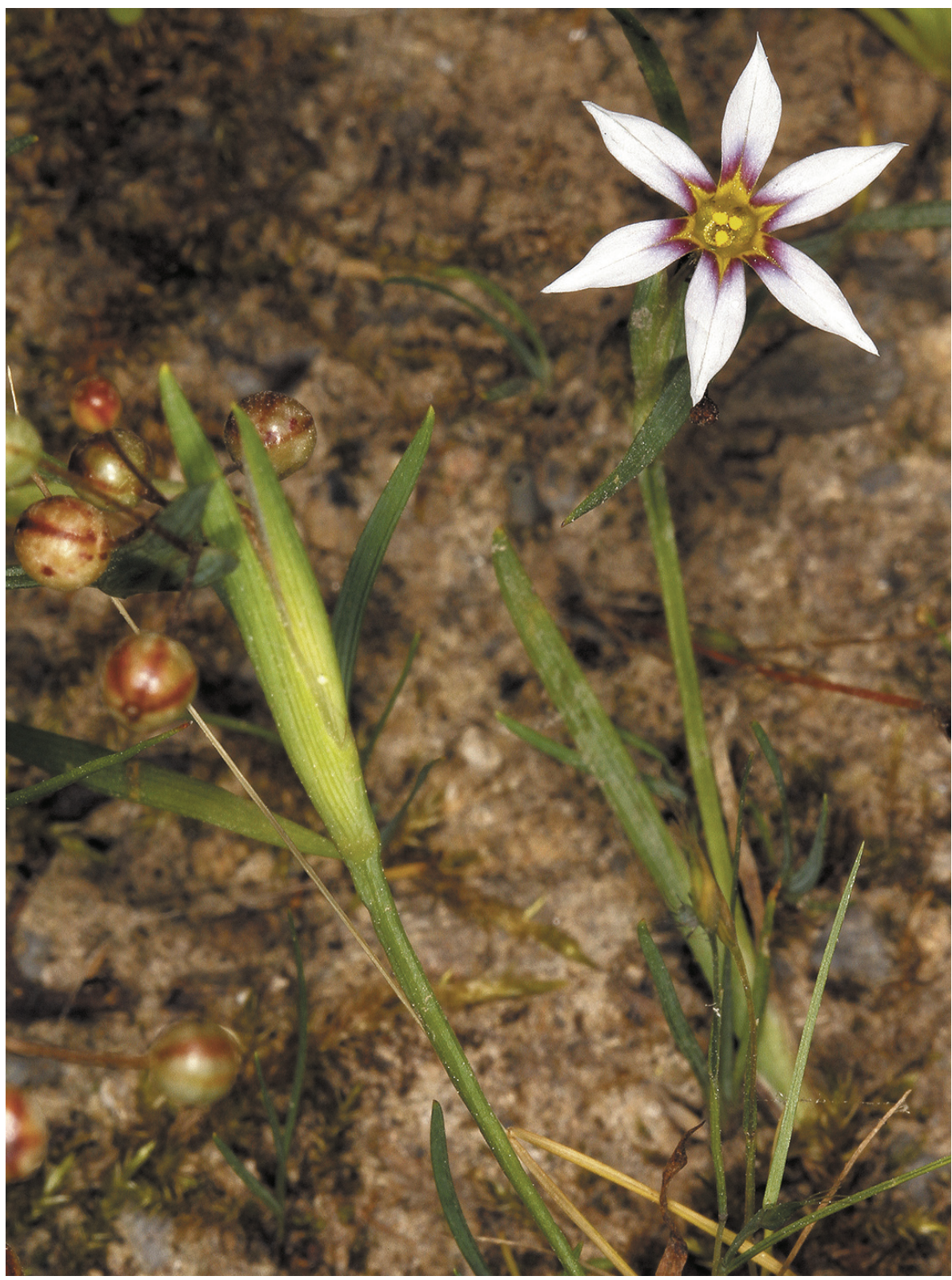

Fig. 10.- Sisyrinchium rosulatum, Monte Mello (Muskiz) 


\section{Conclusiones}

A modo de conclusión queremos destacar las nuevas citas provinciales: Viola cornuta para Burgos, si bien muy cerca del límite con Cantabria; y Tripleurospermum inodorum y Sisyrinchium rosulatum, ambas para Vizcaya, esta última proveniente de Norteamérica y que está empezando a aclimatarse en los ámbitos termófilos del occidente vizcaíno, al igual que otras plantas neófitas, como parecen ser Onobrychis viciifolia y Melilotus segetalis; o arqueófitos como Linum usitatissimum.

Además destacar las citas de algunas poblaciones que, como en trabajos anteriores, parecen traslucir cierto efecto pedinótico, como serían, en el presente artículo, Convallaria majalis, Galium verum subsp. verum, Gymnocarpium dryopteris, Lilium pyrenaicum y Xiphion latifolium, entre otras.

\section{Agradecimientos}

A Carlos Melgosa, Ma Ángeles Bernaola y Manu Franco, por su siempre desinteresada colaboración; y a Javier Benito Ayuso.

\section{Bibliografía}

- Aedo, C., Aldasoro, J. J., Argüelles, J. M., Carlón, L., Díez Riol, A., Gómez Casares, G., González Del Valle, J. M., Guillén Oterino, A., Laínz, M., Moreno Moral, G., Patallo, J., Sánchez Pedraja, Ó. 2001. Contribuciones al conocimiento de la flora cantábrica, V. Bol. Cien. Nat. R.I.D.E.A. 47: 7-52.

- Aedo, C. 2003. Observaciones sobre la flora cantábrica. Memoria inédita. Madrid.

- Aizpuru, I., Aperribai, J.A., Garin, F. 1989-1990. Araba, Bizkaia eta Gipuzkoako Landare katalogoari eraskin eta zuzenketak (II). Munibe, Cienc. nat. 41: 123-126.

- Aizpuru, I., Aseginolaza, C., Garin, F. , Vivant, J. 1998. Contribuciones al conocimiento de la flora del País Vasco, III. Munibe, Cienc. nat. 50: 7-19.

- Aizpuru, I., Aseginolaza, C., Uribe-Echebarría, P.M., Urrutia, P., Zorrakin, I. 1999. Claves ilustradas de la flora del País Vasco y territorios limítrofes. Gobierno Vasco. Vitoria-Gasteiz.

- Aizpuru, I., Aperribay, J.A., Garín, F., Garmendía, J., Olariaga, I. 2005. Contribuciones al conocimiento de la flora del País Vasco VIII. Munibe, Cienc. nat. 56: 75-81.

- Alejandre, J.A., Aseginolaza, C., Gómez, D., Montserrat, G., Morante, G., Uribe-Echebarria, P. M., Urrutia, P. , Zorrakin, I. 1987. Adiciones y correcciones al Catálogo florístico de Álava, Vizcaya y Guipúzcoa. Munibe, Cien. nat. 39:123-131.

- Alejandre, J.A., Benito, J., García-López, J.M., Mateo, G. (Eds.) 2014. Actualización del catálogo de la flora vascular silvestre de Burgos. Estado de conocimiento en el invierno-primavera 20132014. José Luis Benito Alonso. Jaca. (Monografías de Botánica Ibérica, 12). 
- Alejandre, J.A., García, J.M. ,Mateo, G. (Eds.) 2006. Atlas de la flora vascular silvestre de Burgos. Junta de Castilla y León, Caja Rural de Burgos. Burgos.

- Allorge, P. , Allorge, V. 1941. Plantes rares ou intéressantes du Nord-Ouest de l'Espagne, principalement du Pays Basque. Bull. Soc. bot. Fr., 88: 226-254.

- Anthos. 2014. Sistema de información de las plantas de España. Real Jardín Botánico, CSIC- Fundación Biodiversidad. Disponible en: www.anthos.es (Consulta realizada en 2013 y 2014).

- Aparicio, J. M., Patino, S., Pérez Dacosta, T., Uribe-Echebarria, P. M., Urrutia, P., Valencia, J. 1994. Notas corológicas sobre la flora vascular del País Vasco y aledaños (VII). Est. Mus. Cienc. Nat. de Álava 8: 85-99.

- Aseginolaza, C., Gómez, D. 1988. Variación del espectro corológico en la transición cántabromediterránea del País Vasco. En: Homenaje a Pedro Montserrat: 391-403.

- Aseginolaza, C., Gómez, D., Lizaur, X., Montserrat, G., Morante, G., Salaberria, M.R., UribeEchebarria, P. M. 1985. Catálogo florístico de Álava, Vizcaya y Guipúzcoa. Gobierno Vasco. Vitoria-Gasteiz.

- Biurrun, I., López Quintana, A. 1991. Notas breves de Botánica. Aportaciones al catálogo florístico del País Vasco (I). Est. Mus. Cienc. Nat. de Álava 6: 71-72.

- Biurrun, I., Campos, J.A., García Mijangos, I., Herrera, M., Loidi, J. 2011. Nuevos datos sobre los bosques de barrancos y pies de cantil (Tilio-Acerion) del País Vasco y regiones limítrofes. Actes del IX Col-loqui Internacional de Botánica Pirenaico-Cantábrica a Ordino. Andorra: 67-74.

- Braun-Blanquet, J. 1967. Vegetationsskiezen aus dem Baskenland mit Aublichen auf das Weitere Ibero-Atlantikum. II Teil. Vegetatio 4: 1-126.

- Bubani, P. 1901. Flora Pyrenaea per Ordines Naturales gradatim digesta. Volumen Quartum. Milán.

- Cadiñanos, J.A., Llorente, A., Fidalgo, E. 2005. Aportaciones a la flora vascular de Vizcaya y Cantabria. Munibe, Cienc. nat. 56: 41-48.

- Cadiñanos, J.A., Fidalgo, E., Llorente, A. 2010. Aportaciones a la flora vascular de Vizcaya, Guipúzcoa, Cantabria y Asturias (III). Munibe, Cienc. nat. 58: 31-38.

- Campos, J.A., Berastegi, A., Darquistade, A. 2002. Sobre algunas plantas poco conocidas del País Vasco y zonas limítrofes. Est. Mus. Cienc. Nat. de Álava 17: 125-130.

- Campos, J.A., Herrera, M. 2009. Diagnosis de la flora alóctona invasora de la CAPV. Dirección de Biodiversidad y Participación Ambiental. Departamento de Medio Ambiente y Ordenación del Territorio. Gobierno Vasco. Bilbao.

- Carlón, L., Laínz, M., Moreno Moral, G., Rodríguez Berdasco, J.M., Sánchez Pedraja, O. 2013: Contribuciones al conocimiento de la flora cantábrica, IX. Documentos del Jardín Botánico Atlántico (Gijón) 10: 1-155.

- Castroviejo, S. [et al.] (Eds.). 1980-2014. Flora Iberica. Plantas vasculares de la Península Ibérica e Islas Baleares. C.S.I.C. Real Jardín Botánico. Madrid.

- Catalán, P., Aizpuru, I. 1985. Aportación al catálogo florístico de la cuenca del Bidasoa (Guipúzcoa y Navarra). Munibe, Cienc. nat. 37: 17-86. 
- Dupont, P. 1964. Herborisations en Espagne Atlantique. I. Biscaye et province de Santander. Monde PI. 343: 6

- Durán, J.A. 2014. Catálogo de la flora vascular de Cantabria. José Luis Benito Alonso, Jaca.

- Fidalgo, E., Llorente, A., Cadiñanos Aguirre, J.A. 2005. Nueva aportación a la corología de la orquídea Epipactis phyllanthes: primera cia para el País Vasco en las dunas de Górliz (Bizkaia). Munibe, Cienc. nat. 56: 157-158.

- Grupo Botánico Cantábrico (GBC). 2014. Index of Orobanchaceae. Disponible en: http://www.farmalierganes.com/flora/angiospermae/orobanchaceae/orobanche/Orobanche_Grex_Minores/Orobanche_amethystea/Orobanche_amethystea.htm.

- Guinea, E. 1949. Vizcaya y su paisaje vegetal (Geobotánica vizcaína). Junta de Cultura de Vizcaya. Bilbao.

- Guinea, E. 1953. Geografía botánica de Santander. Santander.

- Herrera, M. 1995. Estudio de la vegetación y flora vascular de la cuenca del río Asón (Cantabria). Guineana 1: 1-435. Universidad del País Vasco.

- Herrera, M. \& Campos, J.A. 2010. Flora alóctona invasora en Bizkaia. Instituto para la Sostenibilidad de Bizkaia, Bilbao.

- Instituto Alavés De La Naturaleza/Arabako Natur Institutoa. 2014. Herbario digital Xavier de Arizaga. Disponible en: http://www.ian-ani.org/herbario.

- Laínz, M. 1960. Aportaciones al conocimiento de la flora cántabro-astur IV. Bol. Inst. Estud. Asturianos, Supl. 1: 3-42.

- Llorente, A., Cadiñanos, J.A., Fidalgo, E. 2009a. Aportaciones a la flora vascular de Vizcaya, Guipúzcoa y Cantabria. Munibe, Cienc. nat. 57: 47-66.

- Llorente, A., Cadiñanos, J.A., Fidalgo, E. 2009b. Aportaciones a la flora vascular de Burgos. Munibe, Cienc. nat. 57: 67-74.

- Llorente, A., Fidalgo, E., Cadiñanos, J.A. 2010. Aportaciones a la flora vascular de Álava, Burgos, Navarra y Soria. Munibe, Cienc. nat. 58: 39-45.

• Loidi, J., Fernández-Prieto, J.A., Herrera, M., Bueno, A. 2014. La vegetación de la comarca burgalesa de Espinosa de los Monteros. Guineana 20: 1-139.

- Loidi, J., Biurrun, I., Herrera, M. 1997. La vegetación del centro-septentrional de España. Itinera Geobotanica 9: 161-618.

- López, J., Devesa, J.A. 2010. Una nueva combinación en Asteraceae. Acta botánica Malacitana, 35: 213.

- Onaindia, M. 1986. Ecología vegetal de las Encartaciones y Macizo del Gorbea (Vizcaya). Universidad del País Vasco. Bilbao.

- Patino, S., Valencia, J. 1989. Nuevas aportaciones al catálogo florístico de la Comunidad Autónoma Vasca. Est. Mus. Cienc. Nat. de Álava 4: 77-84. 
- Patino, S., Valencia, J., Miguel, E., Prieto, A., Elorza, J., Oyanarte, T., Toral, R., Otxoa, E., Diaz, E. 2009-2010. Notas corológicas sobre la flora vascular del País Vasco y aledaños (XII). Est. Mus. Cienc. Nat. de Álava 23: 91-111.

- Pérez De Ana, J.M. 2004. Nuevas citas de flora amenazada y escasa en Las Encartaciones (Oeste del País Vasco). Est. Mus. Cienc. Nat. de Álava 18-19: 69-79.

- Pérez De Ana, J.M. 2008. Nuevas citas de flora amenazada y rara en Sierra Salvada. Est. Mus. Cienc. Nat. de Álava 22: 85-100.

- Pérez De Ana, J.M. 2010. Nuevas citas de flora amenazada y rara en Sierra Salvada II. Est. Mus. Cienc. Nat. de Álava, 23: 61-66.

- Rico, E. 2013. El género Colchicum (Fam. Liliaceae. Vol. 20). Prueba de imprenta de Flora Iberica. Disponible en: http://www.floraiberica.es/floraiberica/texto/imprenta/tomoXX/20_183_00_ Colchicum_4e.pdf.

- Rodríguez Guitián, M.A. 2010. Temperate riverside forests without alder trees in the northwest of the Iberian Peninsula: ecology, phytosociological profile and interest for preservation policies. Lazaroa 31: 9-37.

- Silván, F., Campos, J.A. 2002. Estudio de la flora vascular amenazada de los arenales de la Comunidad Autónoma del País Vasco. Informe inédito. Dpto. de Medio Ambiente y Ordenación del Territorio.

- Urrutia, P., Zorrakin, I. 1986. Campaña de herborización en Montes de Izkiz. Est. Inst. Alavés Naturaleza 1: 185-221.

- Villar, L., Sesé, J.A., Ferrández, J.V. 1997 y 2001. Atlas de la flora del Pirineo Aragonés, vols. I y ll. Consejo de Protección de la Naturaleza de Aragón-Instituto de Estudios Altoaragoneses. Huesca.

-Willkomm, H.M. 1893. Supplementum Prodromi Florae Hispanicae. Stuttgart.

-Willkomm, H.M., Lange, J. 1880. Prodromus Florae Hispanicae, vol III. Stuttgart.

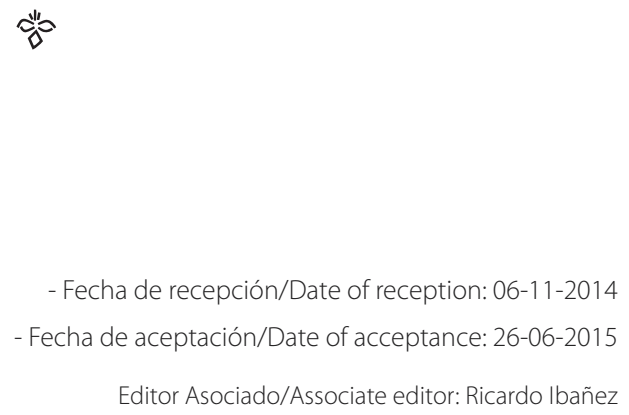

